\title{
Clinical Characteristics and EPS-guided Therapy in 142 Cases of Sustained Ventricular Tachycardia
}

\author{
Katsuya Ebe, MD, Yoshifusa Aizawa, MD \\ and Akira ShIBATA, MD
}

\section{SUMMARY}

One hundred and forty-two consecutive patients with sustained monomorphic ventricular tachycardia (VT) were investigated. Only $26.1 \%$ of VTs were associated with ischemic heart disease (IHD). The induction rate of sustained VT upon electrophysiologic study (EPS) was $82.9 \%$ in patients with IHD and $65.3 \%$ in non-IHD. Of 76 inducible sustained VTs, pharmacologic therapy was finally selected in 35 cases, ablative therapy in 25 and surgical therapy in 12. Long-term prognosis was compared between groups divided according to type of ventricular arrhythmia induced at final EPS after antiarrhythmic therapy as follows: Group A: complete suppression of VT, Group B: clinical or non-clinical nonsustained VT, Group C: clinical sustained VT. The event rate in IHD was $6.3 \%$ in Group A, $44.4 \%$ in Group B and $100 \%$ in Group C. In non-IHD, the event rate was $24.0 \%, 25.0 \%$ and $75.0 \%$ (Groups A, B and C, respectively). Complete suppression of VT showed a good prognosis in IHD, however, a slightly higher recurrence rate was observed in non-IHD. In ablative therapy, some recurrences and sudden deaths were observed in spite of complete suppression of both VTs in both the IHD and nonIHD groups. Review of the efficacy of antiarrhythmic procedures is recommended during the follow-up period. (Jpn Heart J 1996; 37: 73-84)

Key words: Ventricular tachycardia (VT) Electrophysiologic study (EPS) EPS guided therapy Prognosis Long-term follow-up ORE than 300,000 sudden cardiac deaths occur each year in the United
States. ${ }^{1)}$ It is believed that the majority are associated with ventricular dysfunction secondary to previous myocardial infarction which is manifested as ventricular tachycardia (VT). ${ }^{1,2)}$ However, patients with ischemic heart disease are less frequent in Japan and they usually show less severe coronary sclerosis and cardiac dysfunction. ${ }^{3)}$ This seems to be reflected in the population and characteristics of patients who have sustained VT.

In this report, we first present the clinical characteristics of patients with

From the First Department of Internal Medicine, Nïgata University School of Medicine, Niigata, Japan.

Address for correspondence: Katsuya Ebe, MD, Division of Cardiology, Internal Medicine, Nagaoka Red Cross Hospital, Nisseki-cho 2-6-1, Nagaoka 940, Japan.

Received for publication August 10, 1995.

Accepted October 9, 1995. 
monomorphic sustained VT seen in our institute, and second, evaluate the longterm prognosis of antiarrhythmic therapy guided by electrophysiologic study (EPS).

\section{Subjects and Methods}

Study of patients: Between April 1984 and September 1994, EPS was carried out in 142 consecutive patients referred for evaluation of monomorphic sustained VT. The characteristics of the patients are shown in Table I.

Electrophysiologic study: EPS was performed in the nonsedated and postabsorptive state after obtaining written consent from the patient. Our protocol of induction of VT has been previously reported. ${ }^{4-6)}$ Briefly, single and double extrastimuli (triple, if necessary) were given at two basic drives: 600 and 400 msec. Ventricular rapid pacing at a cycle length of 600-286 msec was then performed for 5-15 sec. This electrical stimulation was delivered at two sites of the right ventricle at the apex and the outflow tract. If VT was not induced, isoproterenol was administered to increase the sinus rate by $20 \%$ and the induction protocol was repeated. When VT was not induced in the right ventricle, extrastimuli and rapid pacing were performed in the left ventricle.

Stimuli were given at twice the diastolic threshold and $2 \mathrm{msec}$ of pulse width using a programmable cardiac stimulator (Fukuda Denshi Co., Cardiac Stimulator BC-02A). Intracardiac local electrograms of the ventricle were filtered at 30 and $500 \mathrm{~Hz}$ and recorded simultancously with surface EGG leads I, II, and $\mathrm{V}_{1}$ at a paper speed of $100-200 \mathrm{~mm} / \mathrm{sec}$ (Siemens-Elema, Mingograf 82). In case of a hemodynamically stable VT, overdrive pacing was performed to confirm the criteria of entrainment $t^{5-8)}$ as well as to terminate it. Endocardial mapping was performed to determine the site of VT origin. ${ }^{9-11)}$

Antiarrhythmic therapy: Pharmacologic therapy: When VT was induced in the control state, procainamide was first administered intravenously at a dosage of $600 \mathrm{mg}$. When VT was still inducible, procainamide was added at a dose of up to $1000-1500 \mathrm{mg}$ (usually less than $1000 \mathrm{mg}$ ) and the induction protocol was repeated. Administration of other drugs was as follows: procainamide 2.0-3.0 g/ day (orally: PO), disopyramide $50-100 \mathrm{mg}$ (intravenously: IV) or $300-400 \mathrm{mg}$

Table I. Clinical Characteristics of Monomorphic Sustained Ventricular Tachycardia

\begin{tabular}{llccc}
\hline & & IHD & non-IHD & $p$ value \\
\hline Age & $(\mathrm{yr})$ & $60.8 \pm 10.3$ & $49.7 \pm 16.9$ & $p<0.01$ \\
Male/fernale & $(\mathrm{n})$ & $33 / 4$ & $58 / 19$ & n.s \\
EF & $(\%)$ & $0.41 \pm 0.13$ & $0.52 \pm 0.15$ & $p<0.01$ \\
Syncope & $(\%)$ & 35.1 & 50.1 & n.s \\
\hline
\end{tabular}

Abbreviations are defined in the text. 
(PO), mexiletine 125-250 mg (IV) or 200-400 mg (PO), flecainide 200-300 mg (PO), propafenone 300-450 $\mathrm{mg}(\mathrm{PO})$, cibenzoline 300-450 $\mathrm{mg}(\mathrm{PO})$, aprindine 30-60 mg (PO) and pilsicainide 100-150 mg (PO). Amiodarone was administered at a dosage of $400 \mathrm{mg} /$ day for one week followed by $200 \mathrm{mg} /$ day for another week (PO). In some cases, combinations of Ia and Ib or Ib and II were administered.

Catheter ablation:.5,12-15) Catheter ablation was attempted if patients had drug-refractory VT and surgical intervention was not desirable due to severe cardiac dysfunction or the patient's condition. Direct current ${ }^{5,12,13)}$ (Baxter, Lifepack 7) was used from 1986 to 1988, and radiofrequency currents (Osypka GmbH, Grenzach-Wyhlen, HAT 200 ${ }^{14,15)}$ has been used since 1988 . The site of delivery was the earliest site of activation during $\mathrm{VT}^{10,15)}$ or the site at which pacing resulted in concealed entrainment. ${ }^{16,17)}$ In cases with multiple monomorphic VT, the site of ablation was determined for each QRS morphology. ${ }^{18)}$

Transcoronary chemical ablation was undertaken if the artery supplying blood to the arrhythmogenic substrate was identified. Absoluble ethyl alcohol was given at a dose of $\left.3 \mathrm{ml} \cdot{ }^{19}\right)$

Surgical intervention:20,21) Surgical intervention was carried out exclusively from 1984 through 1989, but catheter ablation has been employed as the first choice since 1989. Surgery was indicated if patients had drug-resistant VT and had no contraindications for open heart surgery. As intraoperative EPS, both endocardial and epicardial mappings were carried out to determine the site of origin of VT. Endocardial resection, myotomy using a cryoablative procedure at $-70^{\circ} \mathrm{C}$ for $2 \mathrm{~min}$, was carried out at the site of origin. The induction protocol was the same as the drug testing mentioned above.

EPS-guided assessment of efficacy of therapies: The efficacy of catheter ablation and surgery was judged by EPS at 2 to 8 weeks after therapy. The protocol was the same as that of VT induction mentioned above. Antiarrhythmic therapy which prevented induction of VT was considered to be effective. VT which was not suppressed by antiarrhythmic agents or not feasible for nonpharmacologic therapies, was followed with antiarrhythmic drugs which had the best effect in reducing the rate of VT.

Long-term follow-up: Patients were followed up in our institution or by the referring physicians. The follow-up data were obtained through telephone contact with patients or by the attending physicians. The duration of follow-up was calculated from the date of the last EPS to the clinical end point: VT recurrence or sudden death. Of the patients who died from nonarrhythmic causes, the duration of follow up was calculated from the last EPS to the date of death. Patients who died perioperatively or died during drug testing were excluded from the long-term follow up. 
Data analysis: Patients were divided into 2 groups according to the underlying diseases: ischemic heart disease (IHD) or non-ischemic heart disease (non-IHD). Clinical and electrophysiologic characteristics were compared between the two groups. Next, according to the results of the final EPS, the patients were subdivided into 3 groups: 1) Group A: sustained VT was not inducible after antiarrhythmic therapy. 2) Group B: clinical VT was prevented, however, nonsustained VT (terminated within $30 \mathrm{sec}$ ) of clinical or nonclinical morphology was induced. 3) Group C: clinical sustained VT was still inducible after drug or interventional therapy, or further therapy was not attempted for medical reasons such as coexisting disorders or cardiac dysfunction. The long-term prognosis was compared among these subgroups.

Continuous data are presented as means \pm S.D. Student's $t$ test and $\chi^{2}$ test were used to assess differences in characteristic findings. A $p$-value less than 0.05 was considered to be significant.

Actuarial survival curves were also estimated using the Kaplan-Meier method in each subgroup A-C of IHD and non-IHD. The Wilcoxon test was used to assess differences in categorial variables of subgroups.

Patients with cathecholamine-sensitive right ventricular tachycardia ${ }^{22)}$ or idiopathic ventricular tachycardia originating from the left ventricle ${ }^{23)}$ were excluded from comparison of inducibility of VT and prognoses.

\section{Results}

Clinical characteristics of sustained VT: Thirty-seven patients $(26.1 \%)$ had IHD. At least one major coronary artery showed significant stenosis or occlusion and previous myocardial infarction was observed in 36 patients. In the remaining patient, the coronary arteries were normal but sustained VTs developed during vasospastic angina.

One hundred-five patients $(73.9 \%)$ had sustained VTs unassociated with IHD and their underlying heart diseases included left ventricular aneurysm without ischemic heart disease (LVan, 8 patients), arrhythmogenic right ventricular dysplasia (ARVD, 14 patients), dilated cardiomyopathy (DCM, 14 patients), hypertrophic cardiomyopathy (HCM, 2 patients), post operative congenital heart diseases (CHD, 7 patients) and miscellaneous heart disease (MHD, 9 patients). MHD included progressive systemic sclerosis, chronic stage myocarditis, sick sinus syndrome, sarcoidosis and mitral prolapse.

No heart disease was observed by routine examination and catheterization in 50 patients. Of these, 17 patients had idiopathic VT characterized by a right bundle branch block and superior axis pattern and responsiveness to verapamil. ${ }^{23)}$ Eleven patients had VT precipitated by exercise. ${ }^{22)}$ Of the remaining 23 patients, 
no heart disease (NO-HD) was observed and VT was not induced by exercise and did not respond to verapamil.

The age of IHD patients was higher than that of non-IHD patients $(60.8 \pm 10.3$ years old vs. $49.7 \pm 16.9$ year old, $p<0.01)$ (Table I). Patients with IHD had significantly lower ejection fraction than that of the non-IHD group $(0.41 \pm 0.13$ vs. $0.52 \pm 0.15, p<0.01)$, however, it was more than 0.3 in all but 2 patients. Of these, the lowest ejection fraction was observed in patients with DCM $(0.36 \pm 0.11)$ and CHD $(0.39 \pm 0.12)$. Syncope was observed more often in the non-IHD patients $(35.1 \%$ vs. $50.1 \%$, n.s.).

Electrophysiologic characteristics of VT: The mean VT rate was slower in the IHD group than in the non-IHD group (183.6 $\pm 37.8 \mathrm{bpm}$ vs. $206.9 \pm 36.7$ $\mathrm{bpm}, p<0.05)$ (Table II). Sustained monomorphic VT was inducible in 76 $(74.5 \%)$ of 102 VTs in the control state. Incessant VT was found at EPS in 7 patients. The remaining 14 patients had VT induced by isoproterenol at EPS or exercise test.

The induction rate was significantly higher in the IHD group than in the non-IHD (82.9\% vs. $63.5 \%, p<0.05)$. However, VTs in "CHD", "ARVD", "LVan" and "DCM" had a high inducibility $(87.5 \%, 81.2 \%, 80.0 \%$ and $75.0 \%$, respectively) (Table II). The lowest induction rate was $26.3 \%$ in "NO-HD" except for idiopathic left ventricular $\mathrm{VT}^{23)}$ and cathecholamine sensitive right ventricular tachycardia. ${ }^{22)}$ Inducibility was unrelated to the cycle length of spontaneous VT.

Table II. Electrophysiologic Characteristics of Monomorphic Sustained Ventricular Tachycardia

\begin{tabular}{llccc}
\hline & & IHD & non-IHD & $p$ value \\
\hline LB/RB/Unknown* & $(\mathrm{n})$ & $10 / 19 / 8$ & $41 / 17 / 19$ & $p<0.01$ \\
Mono/pleo & $(\mathrm{n})$ & $15 / 22$ & $45 / 32$ & $\mathrm{n} . \mathrm{s}$ \\
VT-rate & $(\mathrm{bpm})$ & $183.6 \pm 37.8$ & $206.9 \pm 36.7$ & $p<0.05$ \\
\hline Induction rate & $(\%)$ & 82.9 & 65.3 & $p<0.05$ \\
\hline
\end{tabular}

* = QRS morphology of ventricular tachycardia (left bundle branch block type/right bundle block type/ unknown QRS morphology); ${ }^{+}=$monornorphic/pleomorphic.

Table III. Final Therapy Based on EP-guided Testing

\begin{tabular}{lrc}
\hline & IHD & \multicolumn{1}{c}{ Non-IHD } \\
\hline Drug & 17 & 18 (LVan: 3, ARVD: 8, DCM: 3, HCM: 1, CHD: 1, NO-HD: 2) \\
CA & 7 & 18 (LVan: 2, ARVD: 3, DCM: 2, CHD: 6, MHD: 3, NO-HD: 2) \\
Ope & 4 & 8 (LVan: 3, ARVD: 1, DCM: 1, MHD: 1, NO-HD: 1) \\
Other & 1 & 3 (DCM: 3) \\
\hline Total & 29 & 47
\end{tabular}

Abbreviations are defined in the text. 
Drug-testing: Pharmacologic therapy was selected in $35 \mathrm{VT}$ s of 76 patients with inducible sustained VT (Table III). The mean number of drugs tested was $3.1 \pm 1.4$ (range 1 to 7 ). The efficacious drugs were as follows: Ia drugs in 12 patients (procainamide: $n=7$, disopyramide: $n=4$, cibenzoline: $n=1$ ), Ib drugs in 4 patients (mexiletine: $n=3$, aprindine: $n=1$ ), Ic drugs in 4 patients (flecainide: $n=2$, propafenone: $n=1$, pilsicainide: $n=1$ ), and III drugs in 9 patients (amiodarone: $n=6$, dl-sotalol: $n=3$ ). Combination therapy was efficacious in 3 patients with propranolol and class I drugs (procainamide: $n=1$, disopyramide: $n=2$ ) and in 3 patients with two class I drugs (propafenone + disopyramide, mexiletine + disopyramide and procainamide + disopyramide).

Non-pharmacologic therapy: Among non-responders to the antiarrhythmic drugs, 42 patients underwent either surgical ablation $(n=12)$ or catheter ablation $(n=30)$ (Table III).

Of the 30 patients who underwent catheter ablation, direct currents were applied in 17 cases and radiofrequency currents in 12. In 5 cases (two, direct current, three, radiofrequency), ablative therapy failed and they were treated with additional antiarrhythmic agents.

Chemical ablation with ethyl alcohol was performed in one case in which VT originated from the interventricular septum at the border of an anterior myocardial infarction in whom catheter radiofrequency ablation failed.

Follow-up of patients: Thirty-three of 35 patients were followed with pharmacologic therapy. Sustained VTs were not induced using antiarrhythmic drugs in 20 of 33 cases, however, several ventricular arrhythmias were still inducible in 6 of 33 cases. The remaining 7 VTs were refractory to drugs and had to be treated with antiarrhythmic agents which were effective in slowing the VT rate.

Ablative therapy was attempted in 28 cases. Twenty-one of 28 patients with successful non-pharmacologic therapy were followed: 17 cases without medication and 4 cases with antiarrhythmic agents. In another 7 cases, VTs with multiple morphologies were still inducible in spite of the addition of antiarrhythmic drugs. Eight patients died from arrhythmia before discharge.

Long-term follow-up and data analysis: Of patients in the IHD group, 16, 9, and 1 VT belonged to Groups A, B and C, respectively (Table IV). In non-IHD, 25, 8 and 4 VTs belonged to Groups A, B and C, respectively (Table IV). During the follow-up period of $38.7 \pm 33.8$ ( 1 to 171 ) months, there were 10 recurrences and 7 sudden deaths. IHD had 2 recurrences $(8.0 \%)$ and 4 sudden deaths (16.0\%). Non-IHD had 8 recurrences $(21.6 \%)$ and 3 sudden deaths $(8.1 \%)$.

The event rate in IHD patients was 1 of $16(6.3 \%)$ in Group A and was lower than the other groups: 4 of $9(44.4 \%)$ in Group B, and 1 of $1(100 \%)$ in Group C. There was $1(6.3 \%)$ sudden death in Group A, $2(22.2 \%)$ in Group B, 
Table IV. Prognosis of Monomorphic Sustained Ventricular Tachycardia in EP-guided Therapy.

\begin{tabular}{|c|c|c|c|c|c|c|c|}
\hline & & \multicolumn{3}{|c|}{ IHD } & \multicolumn{3}{|c|}{ Non-IHD } \\
\hline & & $\mathrm{A}$ & B & C & A & $\mathrm{B}$ & $\mathrm{C}\left(^{*}\right)$ \\
\hline No recurrence & $(n)$ & 15 & 5 & 0 & 19 & 6 & 1 \\
\hline Recurrence & $(n)$ & 0 & 2 & 0 & 5 & 1 & 2 \\
\hline Sudden death & $(n)$ & 1 & 2 & 1 & 1 & 1 & 1 \\
\hline Total & $\langle n\rangle$ & 16 & 9 & 1 & 25 & 8 & 4 \\
\hline
\end{tabular}

"A, B and C" represent Groups A, B and C as defined in the text.

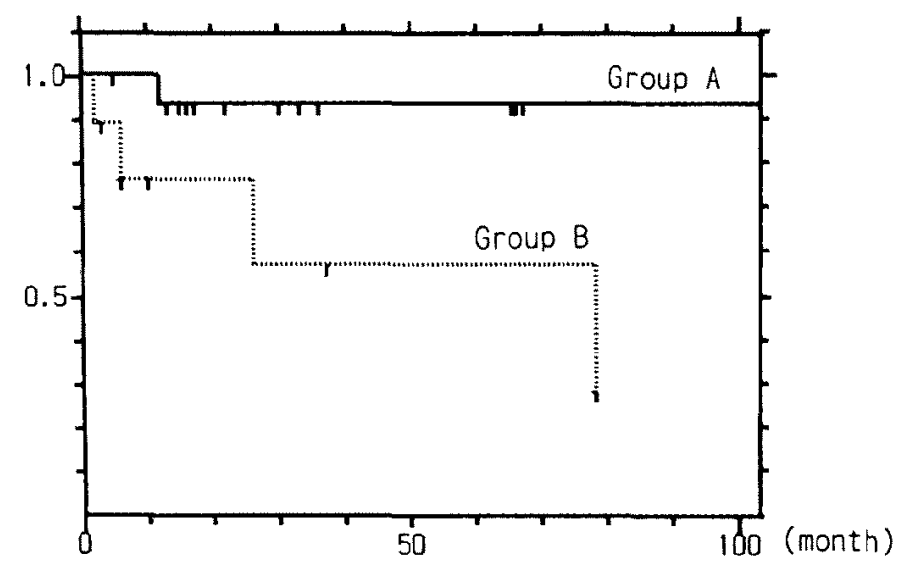

Figure 1. Cumulative incidence rates of events (recurrence and sudden death) in IHD patients. Groups A and B are defined in the text.

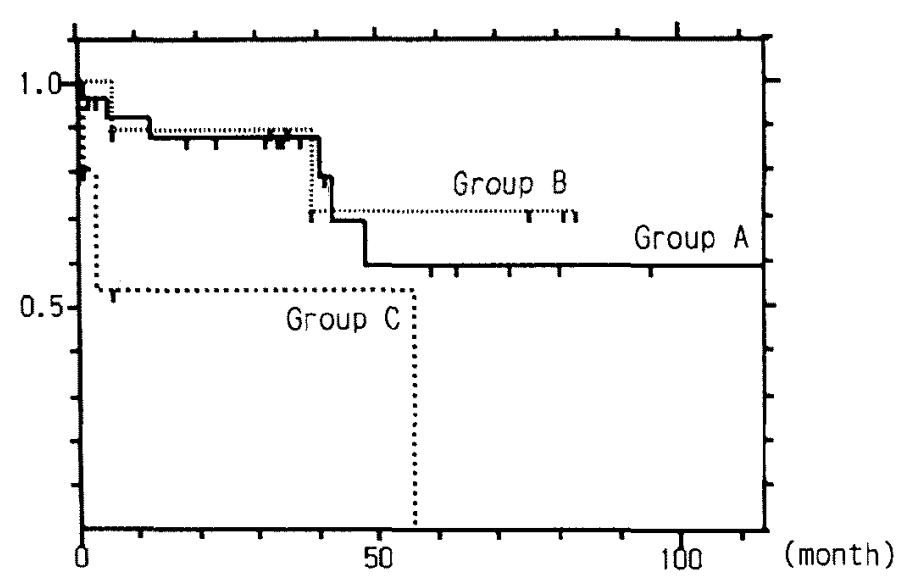

Figure 2. Cumulative incidence rates of events (recurrence and sudden death) in non-IHD patients. Groups A, B and C are defined in the text. 


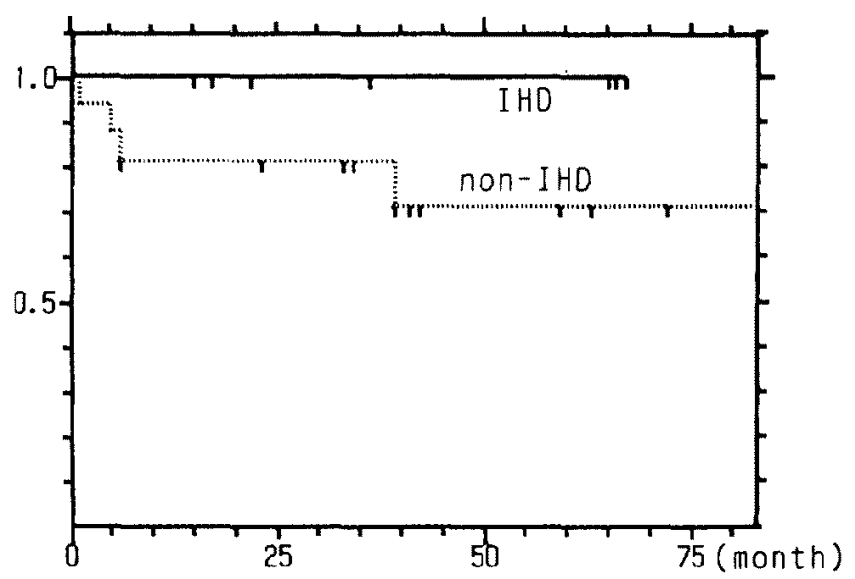

Figure 3. Cumulative incidence rates of events in Group $A$ with antiarrhythmic agents.

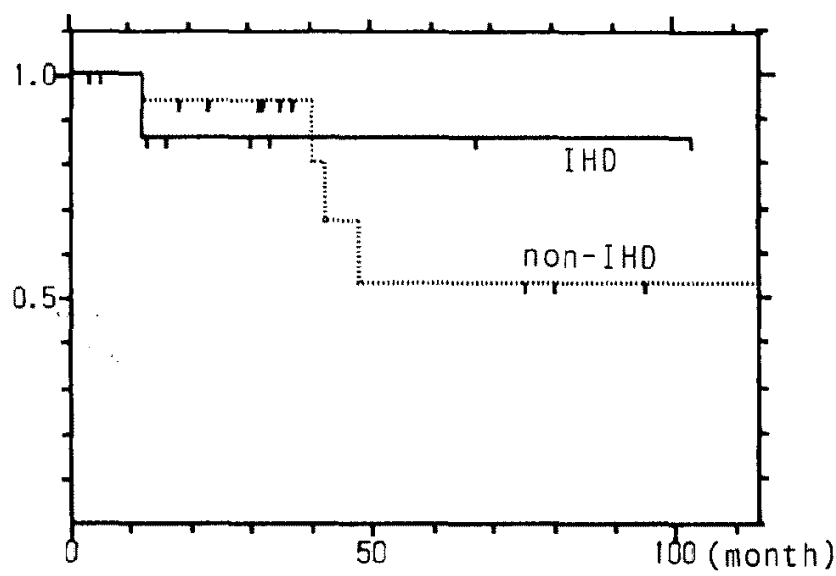

Figure 4. Cumulative incidence rates of events in Group A with ablative therapies.

$1(100 \%)$ in Group C. Using Kaplan-Meier life table analysis in IHD (Figure 1), there were no significant differences in recurrence between Groups A and B ( $p=0.056$ ) because of the small numbers of patients studied within each subgroup. However, Group A of IHD showed better prognoses than Group B. Life table analysis could not be applied to Group C of IHD because it had only one case.

In non-IHD patients, the event rate was 6 of $25(24.0 \%)$ in Group A, 2 of $8(25.0 \%)$ in Group B and 3 of $4(75.0 \%)$ in Group C. Sudden death occured in 1 patient $(4.0 \%)$ in Group A, $1(20.0 \%)$ in Group B, and $1(25.0 \%)$ in Group C. There were also no significant differences in life table analysis between each group (Figure 2), however, Groups A and B showed similar prognoses and better 
prognoses than Group C (A vs. C: $p=0.060$, B vs. C: $p=0.093$ ).

No statistical differences in ejection fraction were observed among the groups for IHD and non-IHD.

Prognoses of patients whose VTs were completely suppressed (Group A) are shown according to the therapies, antiarrhythmic drugs (Figure 3) and ablative therapies (Figure 4). The drug therapy of VT of IHD showed excellent prognosis, however, the non-IHD group showed recurrence in 3 patients. After ablative therapy, VT of IHD also had a better prognosis, however, both IHD and nonIHD groups had a case of sudden death one year after treatment with ablative therapies. There were no significant differences because of the small number of patients.

\section{Discussion}

Clinical and electrophysiologic characteristics of VT: Ischemic heart disease is the most common cardiac disorder in patients with monomorphic sustained VT in Western countries. ${ }^{24)}$ Re-entry is considered to be the underlying mechanism of most monomorphic sustained VTs, and transient entrainment with rapid pacing highly demonstrates strong evidence of re-entry. ${ }^{5-8,16,17)}$ Electrophysiologic characteristics of the re-entrant circuit have been investigated, ${ }^{5-8,16,17,25)}$ however, the efficacy of antiarrhythmic drug therapy is limited and not predictable. ${ }^{26-30)}$

In VT unassociated with non-ischemic heart disease, re-entry is also shown to be the most common underlying mechanism in case of inducible monomorphic sustained VT ${ }^{6)}$ As reported by Naccarelli et al, ${ }^{31)}$ the induction rate and prognoses might be different between VT with and without IHD.

In the present study, only $26.1 \%$ of patients with monomorphic sustained VT had IHD as the underlying heart disease and their cardiac function was slightly better than that of IHD in previous reports. ${ }^{5-8,16,17)}$ Seventy-seven patients $(73.9 \%)$ had VT unassociated with coronary heart disease or no demonstrable heart disease.

Inducibility of VT in IHD was slightly lower in the present study than that reported earlier, ${ }^{31,32)}$ however, this might be partly due to the fact that some patients had incessant VT. The inducibility of non-IHD was as high as that reported by Naccarelli. ${ }^{31)}$

EPS-guided assessment of the efficacy of antiarrhythmic therapies: The efficacy of drugs and ablative therapies has been determined by EPS. ${ }^{26-30}$ ) An optimal stimulation protocol and the end point in EPS guided judgment is, however, still controversial. More aggressive protocols may result in more non-clinical ventricular arrhythmias. ${ }^{32)}$ In some recent studies, less aggressive protocols 
have been used with most patients. ${ }^{29,30)}$

The end point of our antiarrhythmic therapy was suppression of monomorphic sustained VT which had been induced in the control state. ${ }^{24}$ However, ventricular arrhythmias sometimes remain inducible after antiarrhythmic therapies. In this study, we divided the patients according to the result of EPS into three subgroups: Groups A-C and long-term prognoses were analyzed. The change of difficulty in inducing VT was not taken into consideration in this study. ${ }^{26)}$

Long-term follow up: In our study, Group A of IHD showed a $6.3 \%$ recurrence rate (Table IV). In contrast, recurrence and sudden death were observed more frequently in Group B $(44.4 \%)$. Complete suppression of ventricular arrhythmia during acute drug testing in EPS has been reported to predict longterm prognoses. ${ }^{26-28)}$ In VT of IHD, our patients with noninducible ventricular arrhythmias after the anti-arrhythmic procedure also showed good long-term prognosis (Figure 1).

In non-IHD patients, Naccarelli et $\mathrm{al}^{3 !)}$ showed that complete suppression is a predictor of a good prognosis in non-IHD patients. In our study, fewer sudden deaths were observed during the follow-up in Group A, however, a similar recurrence rate was observed in the patients whose VTs were completely suppressed (Group A) and in patients whose non-sustained VTs were still inducible (Group B) (Table IV). Complete suppression of ventricular arrhythmia in EPS may not predict a good prognosis in non-IHD patients (Figure 2). Variable underlying diseases are involved in this study and they are different from Naccarelli's. Progressive degeneration of the myocardium may be one of the reasons why effective agents in the acute phase became ineffective.

Some sudden deaths were observed during the follow-up period in patients whose VTs were treated with ablative therapy and which were completely suppressed in acute EPS guided judgments. Chronic fibrosis in the ablated myocardium may be attributed to such recurrences, however, few studies have reported the electrophysiologic characteristics of the re-entrant circuit in the chronic phase after ablated therapy. Further studies are indispensable. Review of the efficacy is recommended at one year after ablated therapy.

Limitation of study: Amiodarone and dl-sotalol were not commonly used until recently, and a higher efficacy might be expected. However, the overall data would be unchanged even if patients treated with class III drugs had been excluded.

Each group consisted of only a small number of patients so more data is needed.

Conclusion: Complete suppression of VT was associated with a good prognosis, with the best being in IHD patients. In non-IHD, slightly higher rates of 
recurrence or sudden death were observed. Some patients with VTs of both IHD and non-IHD died suddenly one year after being treated with ablative therapy, even if VTs had been completely suppressed in acute EPS guided assessment. It should be mandatory to reassess the efficacy of either intervention during the follow-up period.

\section{ReFERENCES}

1. Meyerburg RJ, Kessler KM, Castellanos A. Sudden cardiac death; structure, function and timedependence of risk. Circulation 1992; 85 (1 Suppl): I-2-10.

2. Hurwitz JL, Josephson ME. Sudden cardiac death in patients with chronic coronary heart disease. Circulation 1992; 85 (1 Suppl): I43-9.

3. Saito M, Fukami K, Hiramori K, et al. Long-term prognosis of patients with acute myocardial infarction: is mortality and morbidity as low as the incidence of ischemic heart diseases in Japan? Am Heart J 1987; 113: 891-7.

4. Aizawa Y, Murata M, Satoh M, et al. Requirement of non-pharmacologic interventions in the treatment of recurrent sustained ventricular tachycardia. Jpn Circ J 1990; 54: 1340-8.

5. Aizawa $\mathrm{Y}$, Niwano $\mathrm{S}$, Chinushi $\mathrm{M}$, et al. Incidence and mechanism of interruption of reentrant ventricular tachycardia with rapid ventricular pacing. Circulation 1992; 85: 589-95.

6. Aizawa $\mathrm{Y}$, Naitoh $\mathrm{N}$, Kitazawa $\mathrm{H}$, et al. Frequency of presumed reentry with an excitable gap in sustained ventricular tachycardia unassociated with coronary disease. Am J Cardiol 1993; 72: 916-21.

7. Waldo AL, Henthorn RW, Plumb VJ, et al. Demonstration of the mechanism of transient entrainment and interruption of ventricular tachycardia with rapid atrial pacing. J Am Coll Cardiol 1984; 3 : $422-30$.

8. Okumura K, Olshansky B, Henthorn RW, et al. Demonstration of the presence of slow conduction during sustained ventricular tachycardia in man: use of transient entrainment of the tachycardia. Circulation $1987 ; 75: 369-78$.

9. Josephson ME, Waxman HL, Cain ME, et al. Ventricular activation during ventricular endocardial pacing. II. Role of pace-mapping to localize origin of ventricular tachycardia. Am J Cardiol 1982; 50: 11-22.

10. Stevenson WG, Weiss $\mathrm{J}$, Wiener I, et al. Localization of slow conduction in a ventricular tachycardia circuit: implications for catheter ablation. Am Heart J 1987; 114: 1253-8.

11. Josephson ME, Horowitz LN, Farshidi A. Continuous local electrical activity. A mechanism of recurrent sustained tachycardia. Circulation 1978; 57: 65965 .

12. Oda $\mathrm{H}$, Aizawa $\mathrm{Y}$, Murata $\mathrm{M}$, et al. A successful electrical ablation of recurrent sustained ventricular tachycardia in postoperative case of tetralogy of Fallot. Jpn Heart J 1986; 27: 421-8.

13. Hartzler GO. Electrode catheter ablation of refractory focal ventricular tachycardia. J Am Coll Cardiol 1983; 2: 1107-13.

14. Chinushi $\mathrm{M}$, Aizawa $\mathrm{Y}$, Kuwano $\mathrm{H}$, et al. Successful radiofrequency current catheter ablation of sustained ventricular tachycardia. Pacing Clin Electrophysiol 1992; 15: 1460-6.

15. Aizawa $\mathrm{Y}$, Chinushi M, Naitoh N, et al. Catheter ablation with radiofrequency current of ventricular tachycardia originating from the right ventricle, Am Heart J 1993; 125: 1269 75.

16. Waldo AL, Henthorn RW. Use of transient entrainment during ventricular tachycardia to localize a critical area in the reentry circuit for ablation. Pacing Clin Electrophysiol 1989; 12: 231-44.

17. Morady F, Kadish A, Rosenheck S, et al. Concealed entrainment as a guide for catheter ablation of ventricular tachycardia in patients with prior myocardial infarction. J Am Coll Cardiol 1991; 17:67889.

18. Josephson ME, Horowitz LN, Farshidi A, et al. Recurrent sustained tachycardia. 4. Pleomorphism. Circulation 1979; 59: 459-68.

19. Brugada $\mathbf{P}$, Swart $\mathrm{H}$, Smeets JL, et al. Transcoronary chemical ablation of ventricular tachycardia. Circulation $1989 ; 79: 475-82$. 
20. Horowitz LN, Harken AH, Kastor JA, Josephson ME. Ventricular resection guided by epicardial and endocardial mapping for treatment of recurrent ventricular tachycardia. N Eng J Med 1980; 302: 589 93.

21. Gallagher IJ, Anderson RW, Kasell J, et al. Cryoablation of drug-resistant ventricular tachycardia in a patient with a variant scleroderma. Circulation 1977; 57: 190-7.

22. Buxton AE, Marchlinski FE, Doherty JU, et al. Repetitive, monomorphic ventricular tachycardia: clinical and electrophysiologic characteristics in patients with and patients without organic heart disease. Am J Cardiol 1984; 54: 997-1002.

23. Ohe $\mathrm{T}$, Shimomura $\mathrm{K}$, Aihara $\mathrm{N}$, et al. Idiopathic sustained left ventricular tachycardia: clinical and electrophysiologic characteristics. Circulation 1988; 77: 560-8.

24. Josephson ME. Recurrent ventricular tachycardia. In Josephson ME, editor. Clinical Cardiac Electrophysiology, Philadelphia: Lea \& Febiger, 1993: 417-615.

25. Aizawa $Y$, Naitoh $N$, Washizuka $T$, et al. Procainamide-induced changes in reentrant ventricular tachycardia with special reference to the tachycardia-interrupting critical paced cycle length during transient entrainment with rapid pacing. Jpn Heart J 1994; 35: 61 1-23.

26. Borggerefe M, Trampisch HJ, Breithardt G. Reappraisal of criteria for assessing drug efficacy in patients with ventricular tachyarrhythmias: complete versus partial suppression of inducible arrhythmias. J Am Coll Cardiol 1988; 12: 140-9,

27. Rae AP, Greenspan AM, Spielman SR, et al. Antiarrhythmic drug efficacy for ventricular tachyarrhythmias associated with coronary artery disease as assessed by electrophysiologic studies. Am J Cardiol 1985; 55: 1494-9.

28. Waller TJ, Kay HR, Spielman SR, et al. Reduction in sudden death and total mortality by antiarrhythmic therapy evaluated by electrophysiologic drug testing: criteria of efficacy in patients with sustained ventricular tachyarrhythmia. J Am Coll Cardiol 1987; 10: 83-9.

29. The ESVEM investigators: The ESVEM trial. Electrophysiologic study versus electrocardiographic monitoring for selection of antiarrhythmic therapy of ventricular tachyarrhythmias. Circulation 1989; 79: 135460 .

30. Mason JW. A comparison of electrophysiologic testing with Holter monitoring to predict antiarrhythmic-drug efficacy for ventricular tachyarrhythmias. N Eng J Med 1993; 329: 445-51.

31. Naccarelli GV, Prystowsky EN, Jackman WN, et al. Role of electrophysiologic testing in managing patients who have ventricular tachycardia unrelated to coronary disease. Am J Cardiol 1982; 50: 16571.

32. Brugada $\mathrm{P}$, Green $\mathrm{M}$, Abdollah $\mathrm{H}$, et al. Significance of ventricular arrhythmias initiated by programmed ventricular stimulation: the importance of the type of ventricular arrhythmia induced and the number of premature stimuli required. Circulation 1984; 69: 87-92. 